\title{
BMJ Open Single-centre retrospective analysis of growth hormone supplementation in IVF patients classified as poor-prognosis
}

\author{
Kevin N Keane, ${ }^{1,2}$ John L Yovich, ${ }^{1,2}$ Anahita Hamidi, ${ }^{1}$ Peter M Hinchliffe, ${ }^{2}$ \\ Satvinder S Dhaliwal ${ }^{3}$
}

To cite: Keane KN, Yovich JL, Hamidi A, et al. Single-centre retrospective analysis of growth hormone supplementation in IVF patients classified as poor-prognosis. BMJ Open 2017;7:e018107. doi:10.1136/ bmjopen-2017-018107

- Prepublication history for this paper is available online. To view these files please visit the journal online (http://dx.doi. org/10.1136/bmjopen-2017018107).

KNK and JLY contributed equally.

Received 6 June 2017

Revised 13 September 2017

Accepted 13 September 2017

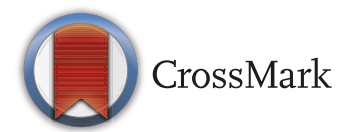

${ }^{1}$ School of Biomedical Science, Faculty of Health Sciences, Curtin University, Perth, Western Australia, Australia ${ }^{2}$ PIVET Medical Centre, Perth, Western Australia, Australia

${ }^{3}$ School of Public Health,

Faculty of Health Sciences, Curtin University, Perth, Western Australia, Australia

Correspondence to Professor John L Yovich; jlyovich@pivet.com.au

\section{ABSTRACT}

Background Patients undergoing in vitro fertilisation (IVF) receive various adjuvant therapies in order to enhance success rates, but the true benefit is actively debated. Growth hormone (GH) supplementation was assessed in poor-prognosis women undergoing fresh IVF transfer cycles.

Methods Data were retrospectively analysed from 400 IVF cycles, where 161 women received GH and 239 did not.

Results Clinical pregnancy, live birth rates and corresponding ORs and Cls were significantly greater with $\mathrm{GH}$, despite patients being significantly older with lower ovarian reserve. Patient's age, quality of transferred embryo and GH were the only significant independent predictors of clinical pregnancy (OR: 0.90, 5.00 and 2.49, $p<0.002$, respectively) and live birth chance (0R: 0.91, 3.90 and $4.75, p<0.014$, respectively). GH increased clinical pregnancy chance by 3.42 -fold $(95 \% \mathrm{Cl} 1.82$ to $6.44, \mathrm{p}<0.0005)$ and live birth chance by 6.16 -fold $(95 \% \mathrm{Cl}$ 2.83 to $13.39, p<0.0005$ ) after adjustment for maternal age, antral follicle count and transferred embryo quality. Conclusion These data provided further evidence to indicate that GH may support more live births, particularly in younger women. It also appears that embryos generated under GH have a better implantation potential, but whether the biological mechanism is embryo-mediated or endometrium-mediated is unclear.

\section{INTRODUCTION}

Many international in vitro fertilisation (IVF) clinics supplement patients with various adjuvant therapies in order to enhance IVF success rates, particularly for those women who are categorised as 'poor prognosis' according to the Bologna criteria. ${ }^{1}$ Some of the most common adjuvant therapies include steroid supplementation, such as dehydroepiandrosterone (DHEA) or testosterone, immune therapy including intravenous immunoglobulin administration and growth hormone (GH) supplementation. ${ }^{2}$ Yet the true beneficial effects of these therapies are actively debated and often only trialled in older patients who are traditionally difficult to treat. ${ }^{2}$ The confusion regarding potential

\section{Strengths and limitations of this study}

One of the largest analyses of the role of growth hormone in in vitro fertilisation over a period of 7.5 years.

- Subject selection was independent and blinded to clinical outcomes.

A retrospective design and observational in nature.

benefits arises from various studies that have demonstrated either inconsistent or opposite findings, used small patient cohorts or have been poorly designed with no blinding or placebo control. As with most fields of medicine, but particularly relevant in the context of IVF, strict double-blind, placebo-controlled, randomised clinical trials (RCTs) are difficult to complete, as demonstrated by the recent early closure of the LIGHT study (Live birth rate In vitro fertilisation and Growth Hormone Treatment), in Australia and New Zealand. ${ }^{3}$ Fully blinded RCTs in IVF are problematic mainly because of patient recruitment issues, where ageing women prefer not to commit several months of their reproductive lifespan to a placebo agent that ultimately may not help them attain pregnancy. Instead, patients tend to opt for any additional treatment, cost permitting, that would potentially help them to fall pregnant. Consequently, while retrospective or observational studies are not 'optimally' designed and rather limited, they still provide important data concerning therapeutic interventions in IVF, especially where sufficiently powered RCTs are lacking, as observed with GH studies.

Since 1988, several trials including observational, sequential crossover and RCTs have been performed to evaluate the clinical benefit of $\mathrm{GH}$ supplementation in patients undergoing IVF. ${ }^{4}$ The first report by Jacob's group in 1988 showed that GH improved ovarian sensitivity to human menopausal gonadotropins in women with 
hypogonadotropic hypogonadism. ${ }^{5}$ Subsequently, several small double-blind placebo-controlled RCTs were initiated, but failed to reveal improvements in ovarian response or clinical parameters, including number of oocytes retrieved and fertilised. ${ }^{6-8}$ In addition, while Busacca $e t$ al found that GH decreased duration of ovarian stimulation, along with reducing follicle stimulating hormone (FSH) dose and concomitantly increasing the number of developing follicles, ${ }^{9}$ other groups such as Levy et al and Suikkari et al observed no such significant change. ${ }^{10} 11$ However, since the mid 2000s, interest in GH as an adjuvant therapy in IVF treatment has been resurrected by several interesting reports.

The study by Tesarik et al showed that GH reduced the number of miscarriages in patients with advanced maternal age (over 40 years) and thus increased the live birth delivery rate. ${ }^{12}$ Interestingly, GH had little effect on pregnancy rates or number of oocytes retrieved in these patients. Similarly, an earlier study from our clinic demonstrated that GH improved live birth rates (20\% vs $7 \%$ ) and reduced miscarriage rates ( $35 \%$ vs $48 \%$ ) in a sequential crossover study. ${ }^{13}$ Increased pregnancy rates for fresh and frozen cycles were also observed. ${ }^{13}$ Conversely, others have shown that although GH can increase oocyte and embryo retrievals, it failed to improve pregnancy rates in poor responders, which adds further complexity to the potential benefits of GH. ${ }^{14}{ }^{15}$ More recently, in a prospective cohort with a concerted effort to reduce the cost associated with GH therapy in IVF, it was found that low dose GH (0.5 IU per day) increased the clinical pregnancy rate in poor responders, while also improving the number of top quality embryos produced. ${ }^{16}$ Taken together, these data indicated that any positive effect from GH may centre on improved embryo and oocyte quality, which may lead to reduced aneuploidy and subsequent miscarriages in poor prognosis or older patients. In the current report, using a new cohort of patients, we examined whether GH improved IVF success rates by reducing miscarriage rates and thereby increasing the delivery rate. Importantly, the study also allowed us to investigate the impact of GH on the number of low or high quality embryos generated, which facilitated the exploration of the potential mechanism by which this adjuvant may exert its benefits.

\section{MATERIALS AND METHODS}

\section{Study period and participants}

This retrospective study investigated all initiated cycles between 1 April 2008 and 31 December 2015 at our clinic. We specifically focused on a subset of patients who had a history of being offered IVF adjuvants by attending clinicians (independent of the researchers), because they were classified as poor-prognosis with one or more of the following criteria: (i) women with fewer than four metaphase II (M II) oocytes although receiving maximal FSH stimulation (ie, 450 IU per day); (ii) women where the majority of embryos were graded poor quality with marked fragmentation $(>50 \%) ;{ }^{17}$ (iii) women with repetitive fresh or frozen embryo transfers (ETs) $(\geq 3$ transfers) without pregnancy; (iv) women aged $\geq 40$ years who had at least one failed IVF cycle. These criteria have been previously published ${ }^{13}$ but have been modified by inclusion of patients with advanced maternal age on the basis of the Bologna criteria. ${ }^{1}$

To offset the bias of individual patients receiving multiple treatment cycles, only the first IVF cycle with successful ovum pick-up (OPU) and fresh ET for each patient within the study period was considered for analysis. In addition, only cycles where no adjuvant therapy or GH-only was administered were included, meaning that cycles with other adjuvant treatment including DHEA and melatonin were excluded in the final analysis. In total, 400 eligible women had 400 IVF cycles that resulted in successful OPU and fresh ET, with 161 cycles/women receiving GH supplementation, (+) GH and 239 cycles/ women free from any adjuvant intervention, designated (-) GH (figure 1). Importantly, patients selected to use $\mathrm{GH}$ on the basis of several factors, one of which was cost (since patients were required to pay).

\section{Clinical management}

GH in the form of Saizen was administered during the preceding menstrual cycle on days 2-3, which included injection of six vials over a 6 week period in the lead-up to OPU and equated to $54 \mathrm{IU}$ over 33-37 days averaging 1.5 IU per day. For SciTropin, $0.3 \mathrm{mg}$ GH was injected daily for 45 days prior to trigger, with patients receiving $\mathrm{GH}$ at precisely 1.0 IU per day up to OPU. All patients were stimulated with recombinant FSH using specific dosage algorithms as defined recently ${ }^{18}$ and in most cases $(49.0 \%$ of cycles) using an antagonist protocol. Other older patients received a flare-agonist regimen $(33.5 \%)$ or specialised downregulation protocols $(17.5 \%)^{19}$ (table 1). Ovulation was triggered with human chorionic gonadotropin (HCG). OPU was undertaken 36 hours post-trigger under intravenous sedation using a double-lumen flushing/ aspiration needle (Cook, Australia). The luteal phase was managed using HCG support. ${ }^{20}$ Additional support hormones were given as required (estradiol, progesterone or combined estradiol/progesterone pessary). Where $\geq 12$ oocytes were recovered, progesterone pessaries replaced HCG injections.

\section{Embryo culture and assessment}

Oocytes were cultured for 4-5 hours postcollection before insemination with motile spermatozoa (100000/ $\mathrm{mL}$ ) for IVF or denuded with hyaluronidase and mature oocytes injected using intracytoplasmic sperm injection (ICSI). Day-3 embryos were graded using a fourpoint system, with half points increments as previously published. $^{21}$ Day-5 embryos were graded using the Gardner scoring system for blastocysts. ${ }^{22}$ Although the clinic has a strong policy of single ET, cases categorised as poor prognosis can receive up to two Day-3 embryos (in 82 cycles $(-) \mathrm{GH}$ and $89(+) \mathrm{GH}$ ) or on a rare occasion, three Day-3 embryos (in one cycle (-) GH and one 


\section{All Initiated Cycles \\ $1^{\text {st }}$ April 2008 to $31^{\text {st }}$ December2015}

$(n=3505)$

All Cycles from Patients who were

offered adjuvants Excluding ( $n=440) . .$.

$(n=1488)$

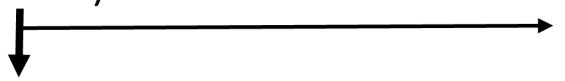

Freeze All Cycles $(n=108)$

Donor Cycles $(n=33)$

Cancelled Cycles ( $n=137)$

Failed Fertilisation $(n=102)$ Failed OPU $(n=49)$

All Cycles with Fresh Embryo Transfer

Ectopic, Blighted Ovum ( $n=11$ )

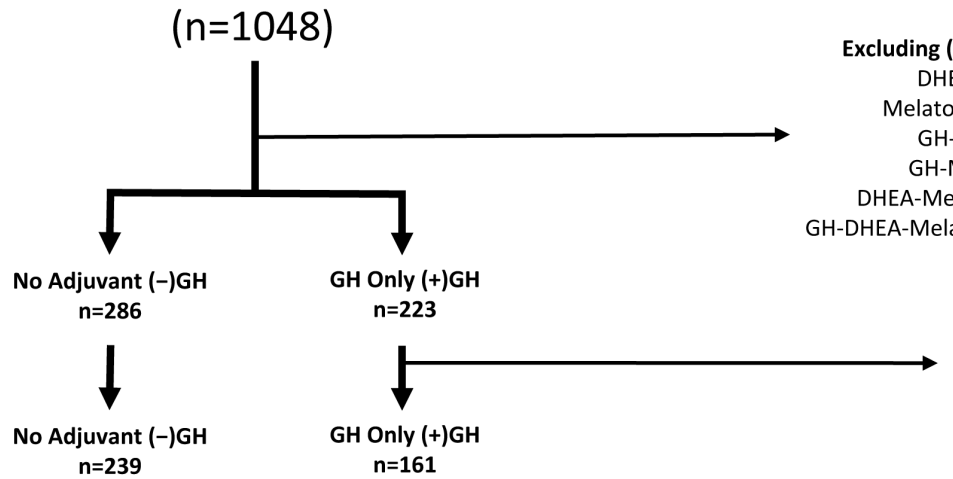

ing $(n=539)$... DHEA Only $(n=68)$

elatonin Only $(n=3)$

GH-DHEA $(n=273)$

GH-Melatonin (21)

EA-Melatonin $(n=30)$

H-DHEA-Melatonin $(n=144)$

Figure 1 Flow diagram of data extraction. Data were extracted from the PIVET database and cases/cycles removed on the basis of cycle outcome (eg, cancelled/donor) and other adjuvant treatment (eg, DHEA/melatonin), cycle type (failed OPU, failed fertilisation or freeze all). To offset the bias of single patients receiving multiple treatment cycles, only the first in vitro fertilisation cycle with successful ovum pick-up and fresh embryo transfer for each patient within the study period was considered for analysis. DHEA, dehydroepiandrosterone; $\mathrm{GH}$, growth hormone.

cycle $(+) \mathrm{GH})$. Blastocysts were transferred in a minority of cycles $(11.7 \%)$.

\section{Data analysis and statistics}

The main clinical outcomes was chance of clinical pregnancy and/or live birth, with the former defined as the presence of an intrauterine gestational sac with foetal heart beat at 7 weeks gestation. Logistic regression was used to assess the independent contributions of individual confounding parameters on these outcomes such as age, body mass index (BMI), anti-Mullerian hormone (AMH) level, antral follicle count (AFC), stimulation protocol type, quality, developmental stage and number of embryos transferred, in addition to the number of patient infertility factors and previous IVF attempts. The unadjusted effect of GH administration on these binary outcomes was also assessed. The effect of each variable was expressed as an OR with associated 95\% CI. Stepwise multiple logistic regression analyses enabled the determination of the minimum number of independent variables that could be used for predicting pregnancy and/ or live birth chance. The coefficients of the independent variables from each of the final logistic regression models were used to calculate OR and CI of pregnancy and/or live birth chance due to the presence or absence of $\mathrm{GH}$. Continuous variables for the (-) $\mathrm{GH}$ and (+) $\mathrm{GH}$ groups were compared using two-sample t-tests and categorical variables were compared using Fisher's exact $\chi^{2}$ tests.

\section{Patient consent and ethical approval}

Our clinic is accredited with the Reproductive Technology Accreditation Committee and the Reproductive Technology Council of Western Australia. These agencies monitor all activities. Specific ethics approval was not required for this study as all procedures and blood tests were embraced by routine approved clinical protocols. However, retrospective analysis and reporting of the data was approved under Curtin University Ethics Committee approval no. RD_25-10. In addition, as part of our documentation system, written and informed consent was obtained from each participant that accepted the use of adjuvants, and they were required to pay for these adjuvants over and above the IVF treatment charges.

\section{RESULTS}

\section{Overview of (-)GH and (+)GH cycle groups}

From the included patient cohort, there was no significant difference between (+) GH and (-) GH cycles with regard to the mean $\mathrm{BMI}$, mean oocytes retrieved, mean two pronuclei generated, fertilisation rate and proportion of high, medium or low quality embryos generated 


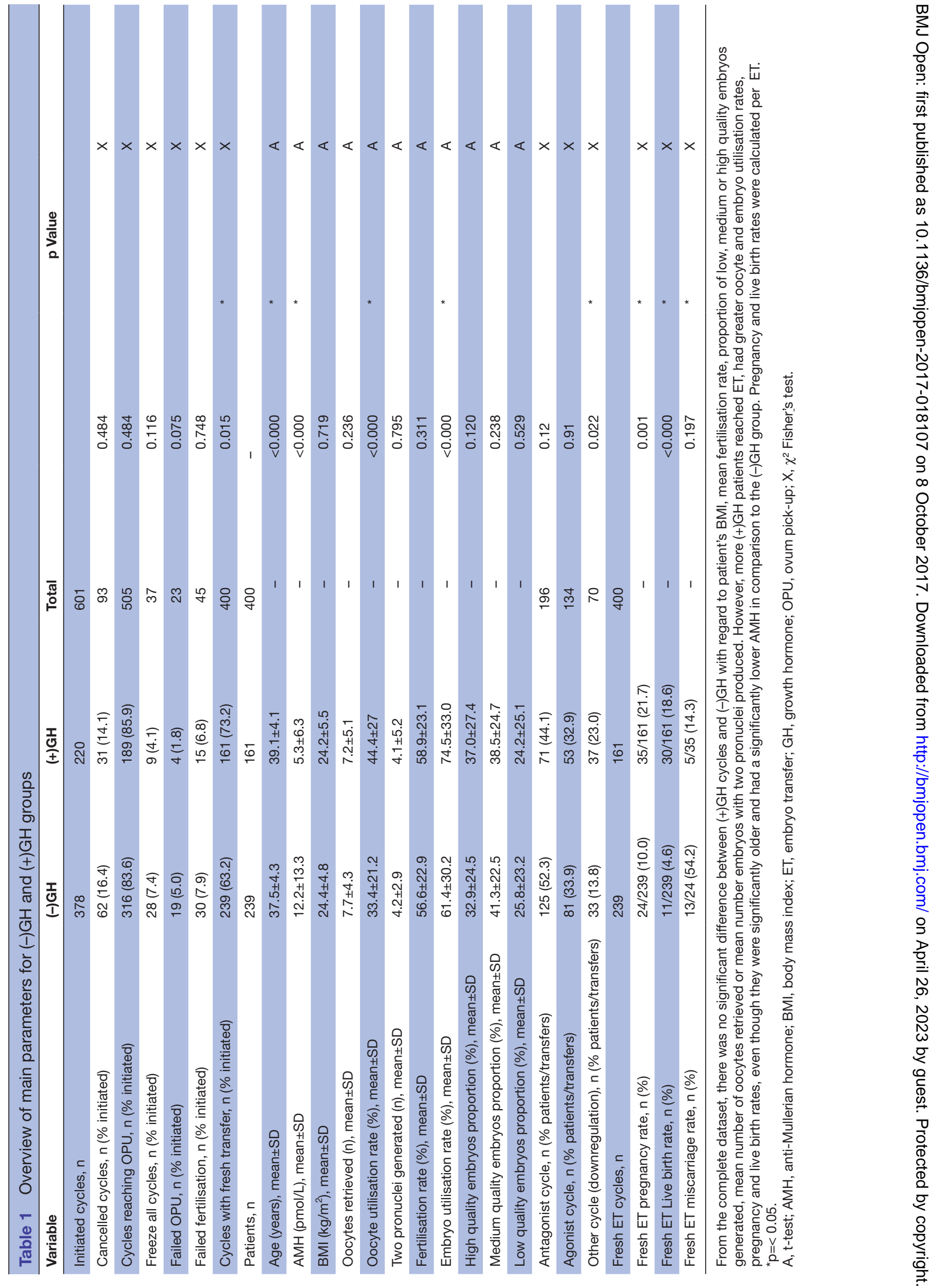


after OPU (table 1). However, the $(+) \mathrm{GH}$ cohort was significantly older (39.1 vs 37.5 years, $\mathrm{p}<0.0002)$, had a lower mean AMH ( 5.3 vs $12.2 \mathrm{pmol} / \mathrm{L}, \mathrm{p}<0.0005)$, but had higher oocyte $(\mathrm{p}<0.0005)$ and embryo $(\mathrm{p}<0.0005)$ utilisation rates (table 1). A significantly higher proportion of (+) GH cycles reached ET ( 73.2 vs $63.2 \%, \mathrm{p}=0.015$ ), while there was no significant difference in the proportion of cycles that were cancelled, with freeze all or resulted in a failed OPU or fertilisation (table 1). However, significantly more $(+) \mathrm{GH}$ cycles were conducted using variations of down regulation ovarian stimulation (23.0 vs $13.8 \%$ ). Nonetheless, (+) GH resulted in significantly higher clinical pregnancy and live birth rates (table 1).

\section{Univariate and multivariate analysis using logistic regression}

Table 2 presents calculated clinical pregnancy and live birth ORs for each individual variable. Only patient's age, AFC, transferred embryo development stage (blastocyst vs cleavage stage), transferred embryo quality and the presence of $(+) \mathrm{GH}$ were significant predictors of clinical pregnancy. All of these variables with the exception of patient's AFC were also predictors for live birth chance. Patient's AMH, BMI, number of embryos transferred, stimulation protocol type, infertility factors or previous IVF attempts did not influence clinical pregnancy and/or live birth chance significantly (table 2). When stepwise multiple logistic regression was performed using all terms, only patient's age, transferred embryo quality and presence of $(+) \mathrm{GH}$ were retained and were all significant. Increasing patient's age decreased the chance of clinical pregnancy and/or live birth by about $11 \%$ per advancing year. When adjusted for patient's age and presence or absence of $(+)$ $\mathrm{GH}$, the chance of clinical pregnancy was increased by 2.24-fold and 7.55-fold when high quality day-3 or high quality blastocysts were transferred, respectively $(\mathrm{p}<0.022)$ (table 2). This increased chance was similar for live birth outcomes (2.01-fold and 3.86-fold, respectively), but was not significant $(\mathrm{p}=0.084$ and 0.073 , respectively). Most importantly, following adjustment for patient's age, AFC and transferred embryo quality, parameters that were critical for outcomes, (+) GH significantly increased the chance of clinical pregnancy success by 3.42 -fold $(95 \%$ CI 1.82 to $6.44, \mathrm{p}<0.0005)$ and significantly increased the chance of live birth success by 6.16-fold (95\% CI 2.83 to 13.39, $\mathrm{p}<0.0005$ ) (table 2).

\section{Interaction of patient's age and (+)GH treatment}

When the data were analysed according to age groups, the effect of $(+) \mathrm{GH}$ was clearly dependent on patient's age. Those aged 35-39 years were 4.50 times more likely to achieve a pregnancy in (+) GH cycles (table 3 ). However, (+)GH did not appear to alter the likelihood of clinical pregnancy significantly in those aged under 35 years or 40 and above, although with higher rates (table 3). Conversely, (+) GH increased the chance of live birth for all patients up to age 44 years. In addition, those under 35 years or between 35 and 39 inclusive, were 3.81 and 14.68 times more likely to achieve a live birth in $(+) \mathrm{GH}$ cycles, respectively (table 3 ). While women aged 40-44 were 5.79 times more likely to achieve a live birth in (+) GH cycles (table 3), further subdivision of this group showed that women aged 40 or 41 years also had an increased chance for live birth (5.42-fold, $\mathrm{p}=0.043$ ), but this was associated with a very wide CI (1.06 to 27.80).

\section{DISCUSSION}

In the current observational study, we showed that patient's age, the quality of transferred embryos and the utilisation of $\mathrm{GH}$, were key predictors of clinical pregnancy and live birth rates in patients undergoing IVF categorised as poor prognosis, due to advanced maternal age, low ovarian reserve makers, previous IVF failure or previous poor quality embryos. Other patient characteristics including BMI, AMH, number of infertility factors or previous IVF attempts did not have an independent effect on clinical pregnancy or live birth chance in this cohort. Patient's AFC also altered pregnancy and live birth OR, with an expected lower chance for those with a low AFC, but it only affected clinical pregnancy significantly. Importantly, following adjustment for patient's age, AFC and quality of the transferred embryo, these data indicated that $(+) \mathrm{GH}$ significantly enhanced the chance of positive pregnancy and birth outcomes, and it was mostly apparent in women aged less than 40 years old. Significantly more live births were observed in the $(+) \mathrm{GH}$ group who had an older average age (mean difference of 1.6years; $37.5(-) \mathrm{GH}$ versus 39.1 years $(+) \mathrm{GH}$ ) and a lower average serum AMH value and consequently could be viewed as a very poor-prognosis group. However, subanalyses also demonstrated a slight but significant live birth benefit in patients who were aged 40 and 41 years, but no effect was observed for pregnancy chance here. Taken together, these data illustrated a clear age-dependent effect from GH supplementation, which appeared to have more positive results in younger IVF patients classified as poor-prognosis . These findings further intensify the debate regarding the potential advantageous effects of GH adjuvant treatment in assisted reproductive technologies, particularly in relation to enhanced live birth rates, the ultimate outcome of IVF success.

These results also echo our earlier work ${ }^{13}$ and comparable data derived from an RCT by Tesarik et al, ${ }^{12}$ which indicated that $\mathrm{GH}$ may reduce aneuploidies, leading to lower miscarriage and higher live births. However, our current dataset contrasts with this RCT where patients had a mean age of 42 years, in that clinical pregnancies and live birth rates were not affected by $(+) \mathrm{GH}$ in our older patient group (above 41 years). This disparity may be due to the difference in the number of transferred embryos in the studies, where on average Tesarik et al transferred 3.5 and 4.2 embryos (-) $\mathrm{GH}$ and $(+) \mathrm{GH}$, respectively, and we transferred 1.36 and 1.58 embryos, respectively. However, number of embryos transferred did not alter clinical pregnancy or live birth chance independently in our study. In terms of oocyte and embryo utilisation rates, these were 
Table 3 The positive effect of GH on clinical pregnancy or live birth chance was clearly dependent on patient's age

\begin{tabular}{|c|c|c|c|c|c|c|c|}
\hline \multirow[b]{2}{*}{ Variable } & $\begin{array}{l}\text { No clinical } \\
\text { pregnancy }\end{array}$ & $\begin{array}{l}\text { Yes clinical } \\
\text { pregnancy }\end{array}$ & Clinical pregnancy & \multirow[b]{2}{*}{ p Value } & \multirow{2}{*}{$\begin{array}{l}\text { Yes live } \\
\text { birth } \\
\text { n (\%) }\end{array}$} & \multirow{2}{*}{$\begin{array}{l}\text { Live birth } \\
\text { OR }(95 \% \mathrm{Cl}) \\
\end{array}$} & \multirow[b]{2}{*}{ p Value } \\
\hline & n (\%) & n (\%) & OR $(95 \% \mathrm{Cl})$ & & & & \\
\hline & \multicolumn{7}{|c|}{ Unadjusted analysis } \\
\hline$(-) \mathrm{GH}, \mathrm{n}(\%)$ & $215(90.0)$ & $24(10.0)$ & 1.00 & - & $11(4.6)$ & 1.00 & \\
\hline \multirow[t]{2}{*}{ (+)GH, n (\%) } & $126(78.3)$ & $35(21.7)$ & 2.49 (1.42 to 4.37$)$ & 0.002 & $30(18.6)$ & 4.75 (2.30 to 9.79$)$ & $<0.000$ \\
\hline & \multicolumn{7}{|c|}{ Analysis according to age group } \\
\hline \multicolumn{8}{|l|}{ Age $<35$ years } \\
\hline$(-) \mathrm{GH}, \mathrm{n}(\%)$ & $47(82.5)$ & $10(17.5)$ & 1.00 & - & $7(12.3)$ & 1.00 & - \\
\hline (+)GH, n (\%) & $15(65.2)$ & $8(34.8)$ & 2.51 (0.84 to 7.50$)$ & 0.100 & $8(34.8)$ & 3.81 (1.19 to 12.24$)$ & 0.025 \\
\hline \multicolumn{8}{|l|}{ Age 35-39years } \\
\hline$(-) \mathrm{GH}, \mathrm{n}(\%)$ & $86(90.5)$ & $9(9.5)$ & 1.00 & - & $2(2.1)$ & 1.00 & - \\
\hline (+)GH, n (\%) & $34(68.0)$ & $16(32.0)$ & 4.50 (1.81 to 11.15$)$ & 0.001 & $12(24.0)$ & 14.68 (3.14 to 68.76$)$ & 0.001 \\
\hline \multicolumn{8}{|l|}{ Age $40-44$ years } \\
\hline$(-) \mathrm{GH}, \mathrm{n}(\%)$ & $78(94.0)$ & $5(6.0)$ & 1.00 & - & $2(2.4)$ & 1.00 & - \\
\hline (+)GH, n (\%) & $69(86.3)$ & $11(13.8)$ & 2.49 (0.82 to 7.51$)$ & 0.106 & $10(12.5)$ & 5.79 (1.23 to 27.3$)$ & 0.027 \\
\hline \multicolumn{8}{|l|}{ Age $>44$ years } \\
\hline$(-) \mathrm{GH}, \mathrm{n}(\%)$ & $4(100.0)$ & $0(0.0 \%)$ & $\mathrm{NC}$ & NC & $0(0.0 \%)$ & $\mathrm{NC}$ & NC \\
\hline (+)GH, n (\%) & $8(100.0)$ & $0(0.0 \%)$ & $\mathrm{NC}$ & NC & $0(0.0 \%)$ & $\mathrm{NC}$ & NC \\
\hline \multicolumn{8}{|c|}{ Age 40 or 41 years only } \\
\hline$(-) \mathrm{GH}, \mathrm{n}(\%)$ & $46(92.0)$ & $4(8.0)$ & 1.00 & - & $2(4.0)$ & 1.00 & - \\
\hline (+)GH, n (\%) & $31(81.6)$ & $7(18.4)$ & 2.60 (0.70 to 9.63$)$ & 0.153 & $7(18.4)$ & 5.42 (1.06 to 27.80$)$ & 0.043 \\
\hline
\end{tabular}

Those younger than 39 year were more likely to achieve clinical pregnancy $(+) \mathrm{GH}$, than $(-) \mathrm{GH}$, but (+)GH did not change the chance for those 40 and older. This was repeated for live birth chance, but those aged 40 or 41 did have a slight but significantly improved chance of live birth $(+) \mathrm{GH}$.

$\mathrm{GH}$, growth hormone; NC, not computed due to low case number.

also elevated $(+) \mathrm{GH}$, but there was no difference in the mean number of oocytes retrieved at OPU or oocytes with two pronuclei generated. Other reports showed that $(+)$ GH increased oocyte and embryo retrieval ${ }^{14}$ and generated more oocytes with two pronuclei. ${ }^{23}$ However, pregnancy and live birth rates were not altered significantly in these studies. ${ }^{14} 23$

Patients with poor prognosis defined by the Bologna criteria have at least two of three clinical parameters which include advanced maternal age ( $>39$ years), a poor ovarian response with three or fewer oocytes collected in a previous cycle or an abnormal ovarian reserve comprising of low AFC $(<7$ follicles $)$ or low AMH $(<8 \mathrm{pmol} / \mathrm{L}){ }^{1}$ Most patients $(32 \%-40 \%)$ in our treatment groups had between 5 and 8 follicles and were graded as AFC category D using our clinical criteria. ${ }^{18} 24$ Furthermore, the $(+) \mathrm{GH}$ group had a significantly reduced serum AMH level and were older on average. In spite of this perceived very poor ovarian reserve and advance maternal age, we are the first to report that $(+) \mathrm{GH}$ improved oocyte and embryo utilisation rates, live births and miscarriage rates in patients with reduced AMH and similarly low AFC ratings. ${ }^{25}$ We also investigated the independent effect of different AFC gradings on successful outcomes and while category $\mathrm{E}(\mathrm{AFC}<5$ follicles) was significantly associated with lower pregnancy rates, this was not observed for live births. Any impact of AFC vanished with adjustment for age indicating it was a weak factor in the current dataset and may reflect our specific rFSH dosage selection algorithms for AFC groups. ${ }^{18}$ However, since patient ovarian reserve has not been described in any IVF study using $\mathrm{GH},{ }^{25}$ direct comparison of our AFC findings with other studies is restricted.

Almost half of the patients in our cohort were stimulated using an antagonist protocol (49.0\%). However, stimulation type did not independently modulate the chance of clinical pregnancy or live birth (data not shown) in these poor-prognosis patients, which follows previous observations. ${ }^{26}$ Furthermore, when adjusting specifically for stimulation protocol, $(+) \mathrm{GH}$ increased clinical pregnancy chance by 2.4-fold $(\mathrm{p}=0.002)$ and live births by 5.0 -fold $(\mathrm{p}<0.0005)$, but again protocol type had no independent impact. Interestingly, other reports have suggested that GH significantly increases the number of embryos transferred in flare agonist cycles, ${ }^{12}{ }^{23}$ but this was observed across all stimulation protocols in our study including antagonist, flare agonist and downregulation ( 1.35 vs $1.59,1.38$ vs 1.64 and 1.36 vs 1.51 embryos transferred $(-) \mathrm{GH}$ and $(+)$ $\mathrm{GH}$, respectively). However, the number of embryos 
transferred did not significantly or independently affect clinical outcomes.

As previously reported, ${ }^{27}$ the quality of the transferred embryo was shown to be a key player in successful pregnancy or live births, and it was confirmed in the current study. Due to the poor-prognosis nature of the patients, the majority of embryos transferred were Day-3 cleavage stage embryos $(88.7 \%)$. Only $11.7 \%$ of transfers used blastocyst culture and consequently, analysis of blastocyst transfer was very limited. Nonetheless, the highest pregnancy and live birth rates were observed when high quality blastocysts or high quality Day-3 embryos were transferred, and these had an independent effect on clinical outcomes.

The authors speculated that GH supplementation might lead to more usable oocytes and embryos, and thus inferring that GH had an impact on egg quality, which has been suggested previously. ${ }^{12}{ }^{13}$ However, when embryo quality was determined using morphological analysis, it was found that GH did not alter the quantity of embryos in low, medium or high quality embryo categories at OPU. Conversely, it was recently shown that low-dose GH was able to slightly but significantly enhance the number of top graded embryos in patients with poor prognosis $(\mathrm{p}=0.04){ }^{16}$ Therefore, it is surprising that this was not demonstrated in the current study. It may be the case that GH supplementation improves embryo quality that cannot be detected through morphological examination.

There are some critical limitations to the current study. As it was observational and retrospective in nature, the potential benefits of GH are mainly associative and not causative. Furthermore, since patients were not randomised, there is potential for patient selection bias. However, we have attempted offset this selection bias by including the first IVF cycle only for each patient within the study period. While the dataset is relatively large, caution must be advised when interpreting the age group subanalysis, as the case number per group was reduced markedly, particularly in relation to the under $35(\mathrm{n}=80)$ and over 44 years $(\mathrm{n}=12)$. Finally, since patients elected to undergo GH treatment and were required to pay for this additional treatment, affordability may also be a significant confounding factor, which was not factored into the current study but may be overcome in future, placebo-controlled studies.

In conclusion, this new observational GH study, the first to include aspects of analysis such as AFC, AMH, BMI and embryo quality assessment, has provided further evidence to indicate the potential beneficial effects of GH supplementation in IVF treatment. Although the study has certain limitations, the data suggested that GH supplementation may provide more live births, mainly in younger women and questions the use of adjuvant therapy in women older than 40 , but particularly over 41 years. While the data do not demonstrate a significant effect on generated embryo quality, it does indicate the (+) GH may lead to more positive outcomes when embryos of lower quality are transferred. This raises the possibility that $\mathrm{GH}$, whose mechanism in IVF is unknown, may not be related to embryo quality, but influences other aspects of female reproduction such as endometrial receptivity.

Acknowledgements The authors thank Curtin University Faculty of Health Science and PIVET Medical Centre for research support. All staff and research participants at PIVET Medical Centre are acknowledged.

Contributors The present work was designed by JLY, KNK and SSD. Data extraction and analysis was performed by KNK, PMH and SSD. The initial manuscript draft was prepared by KNK and subsequently revised by JLY, SSD, AH and PMH. All the authors approved the final submitted version.

Competing interests None declared.

Patient consent Detail has been removed from this case description/these case descriptions to ensure anonymity. The editors and reviewers have seen the detailed information available and are satisfied that the information backs up the case the authors are making.

Ethics approval Human Research Ethics Committee Curtin University.

Provenance and peer review Not commissioned; externally peer reviewed.

Data sharing statement Access to the de-identified dataset may be sought via email request to the lead author JLY.

Open Access This is an Open Access article distributed in accordance with the Creative Commons Attribution Non Commercial (CC BY-NC 4.0) license, which permits others to distribute, remix, adapt, build upon this work non-commercially, and license their derivative works on different terms, provided the original work is properly cited and the use is non-commercial. See: http://creativecommons.org/ licenses/by-nc/4.0/

C Article author(s) (or their employer(s) unless otherwise stated in the text of the article) 2017. All rights reserved. No commercial use is permitted unless otherwise expressly granted.

\section{REFERENCES}

1. Ferraretti AP, La Marca A, Fauser BC, et al. ESHRE consensus on the definition of 'poor response' to ovarian stimulation for in vitro fertilization: the Bologna criteria. Hum Reprod 2011;26:1616-24.

2. Nardo LG, El-Toukhy T, Stewart J, et al. British Fertility Society Policy and Practice Committee: adjuvants in IVF: evidence for good clinical practice. Hum Fertil 2015;18:2-15.

3. Norman RJ, Alvino H, Hart R. For the LIGHT Investigators. A randomised double blind placebo controlled study of recombinant human growth hormone $(\mathrm{r}-\mathrm{HGH})$ on live birth rates in women who are poor responders. Human Reproduction 2016;31:i37.

4. Homburg R, Singh A, Bhide P, et al. The re-growth of growth hormone in fertility treatment: a critical review. Hum Fertil 2012;15:190-3.

5. Homburg R, Eshel A, Abdalla HI, et al. Growth hormone facilitates ovulation induction by gonadotrophins. Clin Endocrinol 1988;29:113-7.

6. Owen EJ, West C, Mason BA, et al. Co-treatment with growth hormone of sub-optimal responders in IVF-ET. Hum Reprod 1991;6:524-8.

7. Bergh C, Hillensjö T, Wikland M, et al. Adjuvant growth hormone treatment during in vitro fertilization: a randomized, placebocontrolled study. Fertil Steril 1994;62:113-20.

8. Dor J, Seidman DS, Amudai E, et al. Adjuvant growth hormone therapy in poor responders to in-vitro fertilization: a prospective randomized placebo-controlled double-blind study. Hum Reprod 1995;10:40-3.

9. Busacca M, Fusi FM, Brigante C, et al. Use of growth hormonereleasing factor in ovulation induction in poor responders. $J$ Reprod Med 1996;41:699-703.

10. Levy T, Limor R, Villa Y, et al. Another look at co-treatment with growth hormone and human menopausal gonadotrophins in poor ovarian responders. Hum Reprod 1993;8:834-9.

11. Suikkari A, MacLachlan V, Koistinen R, et al. Double-blind placebo controlled study: human biosynthetic growth hormone for assisted reproductive technology. Fertil Steril 1996;65:800-5.

12. Tesarik J, Hazout A, Mendoza C. Improvement of delivery and live birth rates after ICSI in women aged $>40$ years by ovarian costimulation with growth hormone. Hum Reprod 2005;20:2536-41.

13. Yovich JL, Stanger JD. Growth hormone supplementation improves implantation and pregnancy productivity rates for poor-prognosis patients undertaking IVF. Reprod Biomed Online 2010;21:37-49. 
14. Eftekhar M, Aflatoonian A, Mohammadian F, et al. Adjuvant growth hormone therapy in antagonist protocol in poor responders undergoing assisted reproductive technology. Arch Gynecol Obstet 2013;287:1017-21.

15. Bassiouny YA, Dakhly DMR, Bayoumi YA, et al. Does the addition of growth hormone to the in vitro fertilization/intracytoplasmic sperm injection antagonist protocol improve outcomes in poor responders? A randomized, controlled trial. Fertil Steril 2016;105:697-702.

16. Lattes K, Brassesco M, Gomez M, et al. Low-dose growth hormone supplementation increases clinical pregnancy rate in poor responders undergoing in vitro fertilisation. Gynecol Endocrinol 2015;31:565-8

17. Yovich JL, Stanger JD, Yovich JM, et al. Quality of embryos from invitro fertilisation. Lancet 1984;1:457.

18. Yovich JL, Alsbjerg B, Conceicao JL, et al. PIVET rFSH dosing algorithms for individualized controlled ovarian stimulation enables optimized pregnancy productivity rates and avoidance of ovarian hyperstimulation syndrome. Drug Des Devel Ther 2016; 10:2561-73.

19. Fisch JD, Keskintepe L, Sher G. Gonadotropin-releasing hormone agonist/antagonist conversion with estrogen priming in low responders with prior in vitro fertilization failure. Fertil Steril 2008;89:342-7.

20. Yovich JL, Stanger JD, Yovich JM, et al. Assessment and hormonal treatment of the luteal phase of in vitro fertilization cycles. Aust $N Z J$ Obstet Gynaecol 1984;24:125-30.
21. Yovich JL, Grudzinskas G. In vitro fertilization and embryo transfer (IVF-ET): current status. The Management of Infertility, a manual of gamete handling procedures, Chapter 10, 1990. Heinemann Medical Books, Oxford. UK (ISBN 043300160 7): 121-44.

22. Gardner DK, Schoolcraft WB. In vitro culture of human blastocysts. Towards reproductive certainty: fertility and genetics beyond 1999. 1999:p. 378-388.

23. Kucuk T, Kozinoglu H, Kaba A. Growth hormone co-treatment within a $\mathrm{GnRH}$ agonist long protocol in patients with poor ovarian response: a prospective, randomized, clinical trial. J Assist Reprod Genet 2008;25:123-7.

24. Keane K, Cruzat VF, Wagle S, et al. Specific ranges of anti-mullerian hormone and antral follicle count correlate to provide a prognostic indicator for IVF outcome. Reprod Biol 2017;17:51-9.

25. Duffy JM, Ahmad G, Mohiyiddeen L, et al. Growth hormone for in vitro fertilization. Cochrane Database Syst Rev 2010: CD000099.

26. Polyzos NP, Nwoye M, Corona R, et al. Live birth rates in Bologna poor responders treated with ovarian stimulation for IVF/ICSI. Reprod Biomed Online 2014;28:469-74.

27. Yovich JL, Conceicao JL, Stanger JD, et al. Mid-luteal serum progesterone concentrations govern implantation rates for cryopreserved embryo transfers conducted under hormone replacement. Reprod Biomed Online 2015;31:180-91. 